\title{
REVISIÓN SISTEMÁTICA ACERCA DE LA ACTIVIDAD FÍSICA Y DE LA SALUD DE POLICÍAS
}

\author{
Rudney da Silva', Cristine Matos's, Beatriz Valdivia ${ }^{3}$, Franciele Cascaes', Paulo Barbosa 5 .
}

${ }^{1}$ Licenciado en Educación Física, PhD. Universidad del Estadio de Santa Catarina, Florianópolis, Santa Catarina, Brasil. ${ }^{2}$ Fisioterapeuta, Master. Laboratorio de Actividad Motora Adaptada. Centro de Ciencias de la Salud y del Deporte. Universidad del Estadio de Santa Catarina, Florianópolis, Santa Catarina, Brasil.

${ }^{3}$ Licenciada en Educación Física, Especialista. Laboratorio de Actividad Motora Adaptada. Centro de Ciencias de la Salud y del Deporte. Universidad del Estadio de Santa Catarina, Florianópolis, Santa Catarina, Brasil.

${ }^{4}$ Fisioterapeuta, Especialista. Laboratorio de Actividad Motora Adaptada. Centro de Ciencias de la Salud y del Deporte. Universidad del Estadio de Santa Catarina, Florianópolis, Santa Catarina, Brasil.

${ }^{5}$ Licenciado en Educación Física, Fisioterapeuta, PhD. Laboratorio de Actividad Motora Adaptada. Centro de Ciencias de la Salud y del Deporte. Universidad del Estadio de Santa Catarina, Florianópolis, Santa Catarina, Brasil.

\section{Correspondencia: rudnex.silva@udesc.br}

Recibido: Abril 1 de 2013 Aceptado: Julio 4 de 2013

\begin{abstract}
Resumen
Este estudio tuvo como objetivo analizar sistemáticamente producciones científicas sobre la actividad física y la salud en policías, publicadas en los últimos cinco años y con indexación en la bases de datos MEDLINE y SCIELO. Fueron realizadas búsquedas en las bases de datos indexadas de BIREME y de PUBMED, disponibles entre los años 2007 y 2012. La búsqueda adoptó los descriptores "actividad física", "salud" y "policial", así como sus correspondientes y también artículos en la lengua española, inglesa y portuguesa. Fueron analizados 24 artículos que presentaban los descriptores adoptados y que cumplían los criterios de inclusión y exclusión. De esta forma, se puede concluir que existe carencia de publicaciones nacionales, principalmente sobre actividad física de policía. También se puede destacar que estudios, tanto nacionales como internacionales, son relacionados con la salud física y mental, principalmente con el estrés, y que son predominantemente no experimentales, y adoptan abordajes cualitativos (nacionales) y cuantitativos (internacionales).
\end{abstract}

Palabras clave: Actividad física, salud, policías.

\section{SYSTEMATIC REVIEW ABOUT PHYSICAL ACTIVITY AND HEALTH OF THE POLICE OFFICER}

\begin{abstract}
This study was aimed to analyze, based on national and international scientific literature, the studies on physical activity and health in Police officers published in the last five years, indexed in MEDLINE and SCIELO databases. Searches in data indexed in PUBMED and BIREME databases, available between 2007 and 2012 period were carried out. The search considered the key words "physical activity", "health" and "police" as well as their related and even in Portuguese, Spanish and English. We reviewed 24 articles that dealt with the descriptors adopted and that met criteria for inclusion and exclusion. So, we can conclude that there is a lack of national publications, mainly on physical activity for police officers. It can be concluded that studies, both national and international, are predominantly no experimental, related to physical and mental health, mainly pertaining to stress, and mostly adopting qualitative (national) and quantitative (international) approaches.
\end{abstract}

Keywords: Physical activity, health, police officers. 


\title{
REVISÃO SISTEMÁTICA SOBRE ATIVIDADE FÍSICA E SAÚDE DE POLICIAIS
}

\begin{abstract}
Resumo
Este estudo teve como objetivo analisar, com base na literatura científica, os estudos sobre atividade física e saúde em Policiais, publicados nos últimos cinco anos e indexados nas bases de dados SCIELO e MEDLINE. Foram realizadas buscas nas bases de dados indexadas na BIREME e na PUBMED, disponíveis entre os períodos de 2007 e 2012 . A busca adotou os descritores "atividade física", "saúde" e "policial", bem como seus correlatos e ainda nos idiomas português, espanhol e inglês. Foram analisados 24 artigos que tratavam dos descritores adotados e que atenderam aos critérios de inclusão e exclusão. Desta forma, pode-se concluir que existe uma carência de publicações nacionais, principalmente sobre atividade física de policiais e ainda que os estudos, tanto nacionais quanto internacionais, são relacionados à saúde física e mental, principalmente quanto ao estresse, são predominantemente não experimentais, de abordagens qualitativas (nacionais) e quantitativas (internacionais).
\end{abstract}

Palavras chaves: Atividade física, saúde, policiais.

\section{Introducción}

La actividad física está directamente relacionada con la promoción de la salud, reducción de las patologías cardiovasculares y la mejora de la calidad de vida. Los agentes que trabajan en seguridad pública son considerados sujetos propensos en desencadenar problemas de salud como patologías cardiovasculares, metabólicas y psíquicas que tienen como causas principales la inactividad y el estrés. Sin embargo, cabe señalar que los estudios sobre la falta de actividad física en la policía todavía son escasos (1-5).

En este contexto, se puede destacar que el bajo nivel de actividad física puede ser considerado como una consecuencia del intenso ritmo de trabajo que afecta principalmente a la participación en las actividades físicas, deportivas y recreativas. Así también, este intenso ritmo de trabajo dificulta la participación en grupos de apoyo psicológico, aumenta la presión para la acción, aumenta las posibilidades de matar y de morir, y aumentan las relaciones no armoniosas entre superiores y subordinados, y que traen como consecuencia un elevado número de licencias para el tratamiento de la salud física y mental de los policías $(3,6-8)$.

Una de las formas de controlar las licencias médicas por los problemas de trastornos mentales, es la práctica de actividades físicas para promover bienestar y equilibrio físico y psicológico en los profesionales. Estudios realizados en las últimas décadas muestran que los policías que no presentan condición física satisfactoria, son propensos a desarrollar enfermedades crónicas degenerativas no transmisibles $(7,9)$. También fueron identificadas escasas publicaciones en relación con la salud de los policías en Brasil (10-16), principalmente en lo que se refiere a los aspectos físicos y psicológicos, a pesar de las preocupaciones actuales sobre seguridad publica.

Se puede señalar que las carencias de estudios sobre las condiciones de inactividad física y la salud de policías en Brasil presentan algunos factores limitadores relacionados a los problemas de seguridad pública, así como las características del estilo de vida y los bajos incentivos a la investigación científica con estos agentes $(2,6,8,17)$. Por lo tanto, teniendo en cuenta la importancia de los agentes de la salud de seguridad pública, los policías en particular, este estudio tuvo como objetivo analizar sistemáticamente producciones científicas sobre la actividad física y la salud en policías, publicados en los últimos cinco años y buscados en la bases de datos MEDLINE y SCIELO.

\section{Materiales y métodos}

El presente estudio es caracterizado como un estudio bibliográfico, que fue llevado a cabo mediante la revisión sistemática de la literatura. El desarrollo de este estudio fue realizado a través de levantamiento, identificación, selección y análisis de la producción científica realizada a través de los siguientes criterios de inclusión: 1) publicaciones en el formato de artículo; 2) publicaciones resultantes de estudios empíricos; 3) publicaciones referentes al período de los años 2007 y 2012; 4) publicaciones disponibles en las bases de 
datos Scientific Electronic Library Online (SCIELO) y Medical Publications of United States National Library of Medicine (MEDLINE/PUBMED).

Fueron adoptados los descriptores de búsqueda en la lengua española, inglesa y portuguesa sobre los siguientes términos "actividad física" (physical activity/ atividade física), "salud" (health, saúde) y "policía" (police officer, policial) y sus correspondientes términos científicos identificados en los Descriptores en Ciencias de la Salud (DeCS), de la Biblioteca Virtual de Salud, de la Biblioteca Regional de Medicina (BIREME), de la Organización Panamericana de Salud y del Gobierno de Brasil, y del Medical Subject Headings (MeSH), de la National Library of Medicine, del National Institutes of Health, de Estados Unidos.

Para los casos de diferencias en la obtención de los resultados, los procedimientos deberían repetirse inversamente por cada autor hasta que fuesen corregidas las discrepancias y así obtener el mismo número de ocurrencias. Después de estos procedimientos, se realizó la selección y el análisis de la producción científica obtenida mediante la adopción de los siguientes criterios de exclusión: 1) artículos de revisión, comentarios, cartas al editor, procedimientos, entre otros; 2) artículos sobre temas no relacionados directamente con la policía, por ejemplo, estadísticas de salud y estadísticas sobre artículos que no presentan los elementos básicos seleccionados para el análisis de la seguridad pública (figura 1); 3) artículos que no presentan los elementos básicos seleccionados para el análisis (figura 1).

Considerando los procedimientos realizados en la figura 1 , fueron seleccionados 24 estudios que fueron incluidos en la revisión.

\section{Resultados}

\section{Descripción de la producción científica brasileña}

Los análisis realizados en los estudios brasileños (2$4,14,15,17-20$ ) sugieren que la mayoría de los policías de las ciudades investigadas presentan indicios de

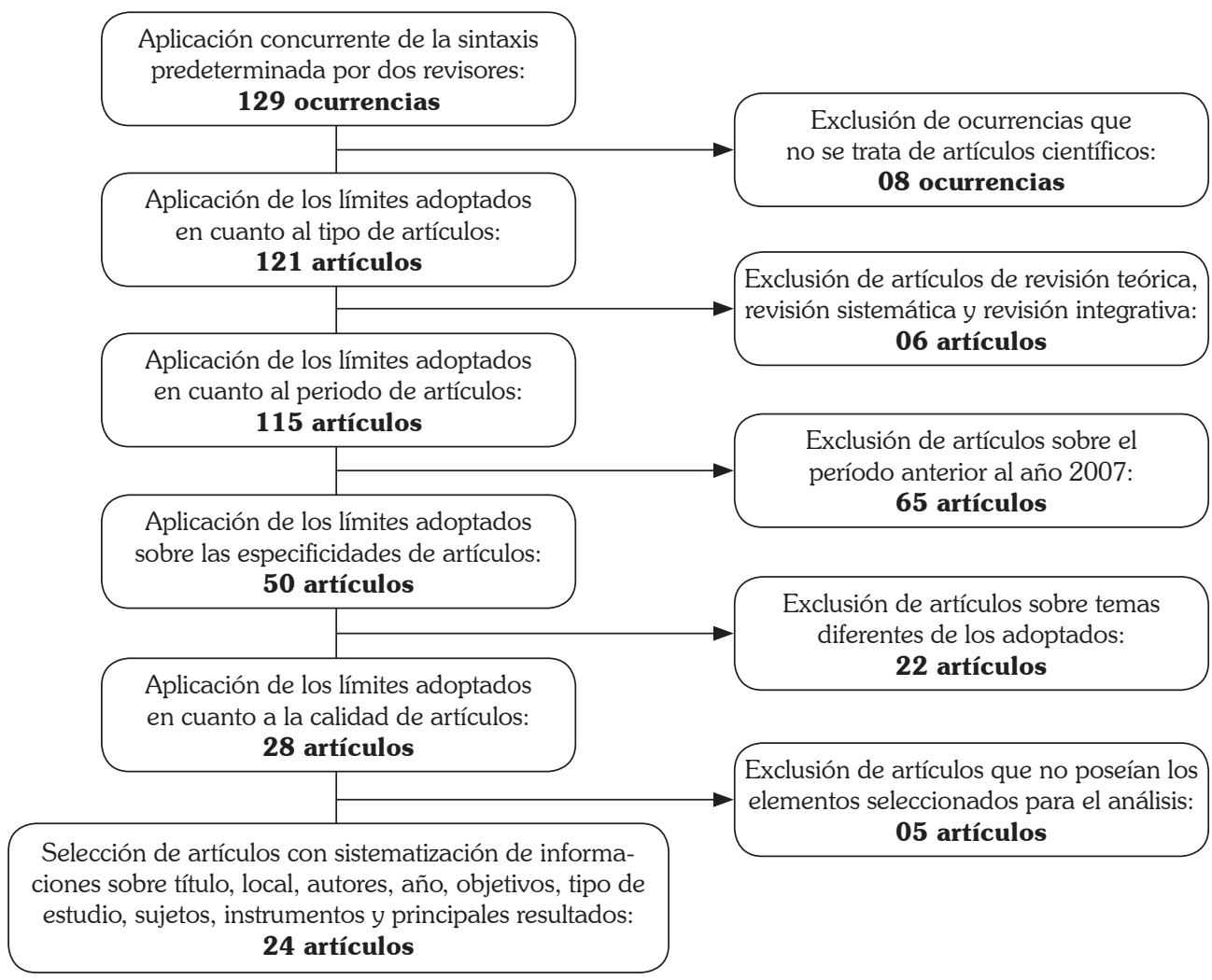

Fuente: Los autores

Figura 1: Flujograma del proceso de selección de artículos 
estrés (sintomatología psicológica y física) en todas las fases de estado (alerta, resistencia, casi-agotamiento y agotamiento). Algunos de los estudios verificaron que intervenciones psicológicas, tanto con policías militares como policías civiles, pueden ser consideradas herramientas positivas en la atención de salud con estas personas, porque existen aspectos organizacionales, ocupacionales y sociales que causan daños a la salud mental, principalmente cuanto al sufrimiento psíquico, alcoholismo, depresión y suicidio.

Los beneficios de estas intervenciones son relacionados principalmente a la mejora de auto-estima, auto-concepto, auto-imagen, percepción de realización profesional, vida personal y profesional. En relación con los aspectos físicos, algunos estudios señalan que los policías militares presentan exceso de peso y escasa frecuencia de actividad física, en cambio, los policías civiles presentan elevados niveles de colesterol, pero los problemas de salud más recurrentes son los dolores de cuello, de espalda, de columna, problemas de visión, dolor de cabeza y jaquecas.

En un estudio de investigación acción con policías civiles de la ciudad de Río de Janeiro/Brasil (2), con objetivo de mostrar los datos relativos a la auto-estima y la calidad de vida de los policías, fue observado, utilizándose del WHOQOL-Bref, Escala de Autoestima de Rosenberg, Escala de Apoyo social y Diagnóstico Organizacional de Krausz, que la intervención mejoró la auto-estima, principalmente el auto-concepto y la auto-imagen, pero no mostró diferencias significativas en la calidad de vida.

En otro estudio de investigación acción también con policías civiles de Río de Janeiro/Brasil (3), en donde se utilizó una estrategia de rescate de autoestima, observando la transformación de la cultura organizacional de la policía a través de una intervención, cuestionario abierto, diario de campo, Escala de Autoestima de Rosenberg y Diagnóstico Organizacional de Krausz, fue verificado que la intervención mejoró la percepción de realización profesional, de auto-imagen, de estrés y de auto-estima. Hubo contribuciones para la vida personal y profesional, principalmente en el aprendizaje en tratar con las adversidades, con la integración de equipo, con la reflexión sobre la calidad de vida en el ámbito personal y profesional y con la minimización del estrés (por el relajamiento y humor).

Estudio empírico, cuantitativo y cualitativo sobre el proceso de enfermedad física y mental de los policías civiles y militares del estado de Rio de Janeiro/Brasil (4), según las condiciones de trabajo y actividades profesionales, utilizándose del instrumento de los tipos cuestionarios, grupos, entrevistas e SRQ-20, fue identificado exceso de peso y escasa frecuencia de actividad física en los Policías Militares, y elevados niveles de colesterol en Policías Civiles. Los mayores problemas de salud fueron el dolor de cuello, dolor en la espalda y columna, los problemas de visón, el dolor de cabeza. En las dos corporaciones de policías se presentó un $16,2 \%$ de daños físicos permanentes, principalmente entre los Policías Militares, que también presentan elevada frecuencia de sufrimiento psíquico.

El estudio cualitativo con policías militares de João Pessoa/Brasil (14) con objetivo de identificar como la Policía Militar se estructura y se relaciona con la salud mental utilizándose observación, investigación documental y entrevista, fue demostrado que el Policía Militar esta en el centro de las fuerzas de la organización del trabajo, de la precarización del trabajo y de las exigencias de la sociedad que contribuyen en acciones dañinas para la salud mental, favoreciendo el aumento de sufrimiento psíquico y pudiéndolo llevar para el alcoholismo, depresión y suicidio.

En el estudio exploratorio y de levantamiento de datos con la participación de la policía militar da ciudad de San Pablo/Brasil (15) sobre la percepción de policías militares de la fuerza táctica y de la calle acerca de los aspectos que se relacionan a su salud mental fue encontrado que la mayoría siempre o algunas veces percibió el estrés, siendo que $41,7 \%$ relata haber hecho alguna acción impulsivamente en determinadas ocasiones; la mayoría siempre o algunas veces se percibió emocionalmente cansado después de un día de trabajo; y otras veces se percibían agresivos en el trabajo; siendo que gran parte pensó en suicidio $(20,8 \%)$ y nunca se pensó realizado con la profesión $(8,3 \%)$.

En un estudio cuantitativo y cualitativo con policías civiles también de la ciudad de Río de Janeiro/Brasil (17), que tenía como objetivo de investigar la existencia del sufrimiento psíquico de acuerdo con diferencias de género, utilizándose el instrumento SRQ-20, fue constatado que no hubo diferencia de sufrimiento psíquico entre los géneros, pero hubo diferencias significativas en algunos ítems de la escala, principalmente entre las Policiales Técnicas.

Un estudio descriptivo y transversal realizado con policías militares de la ciudad de Natal/Brasil (18), sobre 
la ocurrencia y la fase de estrés, y la prevalencia de sintomatología física y mental, fue constatado, utilizando el instrumento Inventario de Síntomas de Estrés para Adultos de Lipp, que la sintomatología de estrés ocurrió en casi la mitad de los policías, presentando síntomas psicológicos (24\%) y síntomas físicos $(24,0 \%)$, siendo $3,4 \%$ en la fase de alerta, $39,8 \%$ en la fase de resistencia, $3,8 \%$ en la fase de casi-agotamiento e $0,4 \%$ en la fase de agotamiento, así como tuvo asociación entre estrés y sexo (mujeres).

En un estudio epidemiológico y transversal con policías militares de la ciudad de Recife/Brasil (19), después de analizar las relaciones entre estilo de vida y aspectos sociodemográficos y ocupacionales a través del cuestionario CCEB, CAGE Test y IPAQ, fue identificado que $12 \%$ fuman, $10 \%$ sugieren consumo abusivo de bebidas alcohólicas, $73 \%$ son insuficientemente activos, $40 \%$ presentan conflictos familiares frecuentes o algunas veces. La edad media de 39 años o más, la baja escolaridad, el bajo nivel económico, el tiempo de profesión de 18 años o más, fueran asociados con el estilo de vida con alto riesgo para la salud.

Estudio exploratorio sobre la percepción de los policías civiles del Distrito Federal del Brasil (20), sobre su contexto de trabajo, sus exigencias, así como las vivencias y los problemas físicos, psicológicos y sociales causados por el trabajo a través de un estudio, utilizándose el instrumento Inventario de Trabajo y Riesgos de Enfermedad, fue encontrado que a pesar de no ser evidentes los daños graves para la salud de los policías novatos, existen riesgos de problemas en las estrategias de mediación en relación a los aspectos que provocan las enfermedades.

\section{Descripción de la producción científica internacional}

Los resultados de estudios realizados en varios países $(5,21-34)$ sugieren que la mayoría de los policías son afectados por eventos traumáticos y/o estrés que provocan sufrimiento psicológico, como el uso de medicamentos, tabaquismo, alcohol, angustia, depresión, ansiedad, entre otros. Una pequeña parte de los estudios también hacen referencia a las consecuencias físicas relacionadas a eventos traumáticos $y / o$ estrés como dolor corporal, obesidad, índices hematológicos inadecuados para salud, baja condición física, diabetes, hipertensión, entre otros. Los eventos traumáticos y/o estresantes más recurrentes son los relacionados directamente a su actuación. Como por ejemplo la exposición frente a heridos en accidentes, la búsqueda de víctimas, la manipulación de restos humanos, las grandes posibilidades de matar o herir gravemente y también de ser muerto, También se incluyen los eventos relacionados a los aspectos organizacionales, como el ambiente de trabajo, la sobrecarga de trabajo, la responsabilidad en servicio, entre otros.

En un estudio transversal realizado en la ciudad de Rahata/Índia (5) con objetivo de evaluar los niveles y las fuentes de estrés entre policías y analizar el estrés como un factor de riesgo para hipertensión, diabetes, obesidad y depresión fue demostrado que la mayoría presentó niveles moderados de estrés, destacando la sobrecarga de trabajo y la responsabilidad personal que tuvieron influencias moderadas sobre el estrés. Fueron identificadas relaciones significativas entre el nivel de estrés con exceso de peso, diabetes, hipertensión y depresión. Los instrumentos utilizados fueran evaluación clínica, OSI y HDRS.

En un estudio transversal con policías de la ciudad de Minneapolis/Estados Unidos (21), sobre los niveles elevados de condición física y actividad física y los daños músculo-esqueléticos, fue verificado, utilizándose entrevista, fue constatado que los policías con mayor condición física fueron menos propensos en dolores de espalda y dolor crónico; los policías que practicaban actividad física presentaron cerca de un tercio de chance en el dolor de espalda y menos de la mitad para dolor crónica; y los policías con obesidad tuvieron tres veces más chance de dolor en la espalda que los policías con índice de masa corporal normal.

En estudio transversal con policías municipales de la ciudad de Búfalo/Estados Unidos (22) sobre la asociación entre índices de obesidad con parámetros hematológicos, fue verificado que la edad predominante fue entre 26 y 61 anos, siendo la mayoría de etnia blanca; que las mujeres fumantes tuvieron un conteo más elevado de glóbulos blancos de que las ex-fumantes o de las que nunca fumaron; $y$ que las mujeres tuvieron un conteo de glóbulos blancos parecidos, pero el conteo de plaquetas fue significativamente mayor.

En estudio longitudinal con medidas transversales repetidas realizado con policías de la ciudad de Palmerston/Norte de Nueva Zelandia (23), que tuvo el objetivo de analizar el impacto de las experiencias traumáticas y organizacionales en salud psicológica de Policías, los resultados mostraron que los eventos 
traumáticos y estresantes organizacionales afectan el sufrimiento psicológico; y que el estrés organizacional tuvo un efecto más fuerte, incluido la exacerbación de los síntomas de trastorno de estrés pos-traumático. Fueron utilizados los instrumentos TSS, IES, HSCL-21 y Escala Diaria de Molestias en Policías.

En un estudio de coorte e histórico realizado en la Holanda con bomberos y policías (24), que tuvo el objetivo de examinar la relación entre la exposición en desastre aéreo de Ámsterdam con síntomas físicos entre bomberos y policías, y explorar el papel de los síntomas de estrés pos-traumático (TEPT), fue mostrado que sujetos expuestos a los desastres aéreos presentan múltiples síntomas físicos causados principalmente por exposición de las víctimas heridas, búsqueda de víctimas o restos humanos, y ocurriendo independientemente de los síntomas de TEPT encontrados.

En otro estudio longitudinal con medidas transversales repetidas (25) también realizado en la Holanda con policías, fueron estabelecidos estandarizaciones sobre la administración del test de evaluaciones de la capacidad de ejecución de demandas físicas de tareas fundamentales del trabajo Policial.

En estudio cualitativo experimental realizado en la ciudad de Londres/Inglaterra (26) para policías y agentes civiles, que tuvo como objetivo determinar los efectos de la condición física pre-lesión sobre la gravedad inicial y de recuperación de lesiones de cuello entre tres y seis meses, los resultados apontan que la recuperación precoz de daños en el cuello es más probable en individuos con niveles medios y altos de condición física de antes de los daños de lo que en individuos con bajos niveles de condición física de antes de los daños. Fueron utilizados los siguientes instrumentos PAS, PP y NDI.

En el estudio con reclutas de la ciudades de Nueva York, Oakland, San Francisco y San José (27), todas de los Estados Unidos, que tuvo como objetivo analizar las relaciones entre histórico familiar de disturbios psiquiátricos con el uso de substancias para los síntomas de estrés pos-traumático, fueron utilizados varios instrumentos (SCID I-NP, SCL-90-R, GSI, FHS, LSC-R, MAST, CIHQ, PCL-S y PDI). Como resultado final se demostró que el histórico familiar para trastornos de humor y ansiedad tuvo un efecto indirecto sobre síntomas de estrés pos-traumático ocurridos en 12 meses, que fueron medidas por el sufrimiento durante el trauma auto-identificado como el peor incidente crítico. El histórico familiar para el uso de substancias también predijo síntomas de estrés pos-traumático después de 12 meses y esta relación fue medida a través de la angustia durante el trauma.

En un estudio de investigación de prontuario desarrollado con policías federales de la ciudad de Viena/ Austria (28) que tuvo como objetivo examinar la tasa de suicidio y características de suicidio entre los Policías de la Policía Federal austríaca, se demostró que las tasas de suicidio entre los policías son comparables con la de la población general de acuerdo con la edad pero, los policías presentan elevado riesgo de suicidio en comparación con trabajadores que tienen salud.

En otro estudio cualitativo transversal desarrollado en la ciudad de Glasgow/Escócia (29) con objetivo de investigar la percepción de los policías sobre sus funciones en las relaciones personales frente a problemas de salud mental y los servicios de salud mental, fue constatado, a través de una entrevista, que las experiencias personales y profesionales de policías sobre personas con trastorno mental en Escocia, tienen en común con los de EUA y las de Australia, ya que el desarrollo de abordajes más colaborativas entre la policía y los prestadores de servicios de salud mental podría ser benéfica.

Estudio transversal de corte prospectivo realizado en los Estados Unidos y Canadá (30) con objetivo de cuantificar la asociación entre el riesgo de disturbio del sueño y de la auto-evaluación sobre salud, seguridad y desempeño de policías, utilizándose los instrumentos Cuestionario Berlín, Insomnio Escala Atenas, RLS, Cuestionario de Cataplejía y ESS, fue encontrado que los disturbios del sueño son comunes entre los policías norte-americanos y presentan asociación significativa con el riesgo elevado de auto-evaluación de salud adversa y el desempeño en seguridad.

Una pesquisa de prontuario y de base de datos desarrollado en la ciudad de Victoria/Austrália (31) con objetivo de explorar la salud mental y las historias de fatalidad en los casos de uso y abuso fatal de fuerza de la Policía entre noviembre de 1982 y febrero de 2007, fue constatado que las muertes provocadas por uso de la fuerza policial, pueden provocar trastornos mentales graves de psicosis y esquizofrenia entre policías.

El estudio transversal con policías civiles y reclutas de la ciudad de Nueva York/EUA (32) sobre la relación 
entre matar o herir gravemente alguien en servicio y los síntomas de salud mental, realizado a través de varios instrumentos (BDHI, $\mathrm{CIHQ}, \mathrm{MCSeCV}, \mathrm{MAST}$ y SASeSR), fue observado que casi $10 \%$ de los policías relataron haber matado o herido gravemente alguien en cumplimiento de su deber en los primeros tres años de servicio. La exposición de riesgo de muerte fue significativamente asociada con síntomas de TEPT y relativamente asociado con síntomas de depresión.

Otro estudio realizado com los oficiales del Departamento de Policía de Milwaukee/EUA (33) tuvo como objetivo utilizar la triangulación de datos para informar las intervenciones para reducir la morbilidad cardiovascular y los factores de riesgo asociados en la policía. Los resultados indicaron que las tasas de sobrepeso $(71,1 \%$ versus $60,8 \%$ ) y la hipertensión $(27,4 \%$ versus $17,6 \%)$ fueron significativamente mayores entre los oficiales que la población general de Wisconsin, siendo que el mayor predictor de enfermedades cardiovasculares (ECV) fue la diabetes.

Otra pesquisa realizada en la Holanda (34), que tenía objetivo de explorar la importancia de la intensidad relativa, duración media y volumen de actividad física en relación con los fatores de risgo de ECV y con síndrome metabólico a través de cuestionario, evaluación física y evaluación antropométrica, fue constatado que la actividad física y la condición física son inversamente asociadas con las anormalidades metabólicas, fue constatado que en lo que respecta a la actividad física, la intensidad específica es la principal característica para determinación de su efecto sobre los factores de riesgo para ECV. Sin embargo, en comparación con la actividad física, la condición física ejerce efectos más acentuados sobre cada uno de los factores de riesgo para patología vascular del corazón individualmente o combinada.

\section{Aspectos metodológicos}

Los análisis de los estudios seleccionados revelaron que hay un total de 24 estudios, nueve estudios nacionales y quince estudios internacionales. Entre los estudios nacionales, la mayoría ( $\mathrm{f}=4$ ) se llevó a cabo en el sureste de Brasil. Algunos de los estudios nacionales ( $f=3$ ) hacen referencia a la investigación con la policía militar y la otra parte se refiere a la investigación con la policía civil $(\mathrm{f}=4)$, y solo un estudio se refiere a la policía militar y civil.
En relación con los estudios nacionales $(\mathrm{f}=9)$, cuatro fueron realizados en Brasil, y en relación con estudios internacionales, cinco fueron desarrollados en los Estados Unidos, tres en Holanda, uno en Estados Unidos y Canadá, y uno en Nueva Zelandia, Australia, India, Inglaterra y Austria. Con base en la clasificación metodología propuesta por Gil (35), se constató que parte de los estudios nacionales e internacionales tienen carácter metodológico, clasificado como: investigación aplicada ( $\mathrm{f}=24$ ), de corte transversal de temporalidad $(\mathrm{f}=12)$, dos nacionales fueron de investigación-acción $y$ dos con abordaje cualitativo.

Todos los estudios nacionales presentaron policías como sujetos de investigación ( $f=7$ ), y de éstos, un estudio se realizó con los registros médicos de los policías. El análisis de los estudios demostró que la mayoría ( $\mathrm{f}=4$ ) adopta un abordaje cuantitativo y es realizado con muestras consideradas medianas y grandes en comparación con el cuantitativo de la población. La mayoría de los estudios internacionales tienen como sujetos exclusivamente policías ( $\mathrm{f}=7$ ) y de éstos, tres estudios se realizaron con policías en formación, denominado reclutas.

En la mayoría de los estudios nacionales $(\mathrm{f}=9) \mathrm{e}$ internacionales ( $f=15$ ) se usaron instrumentos estandarizados para la recopilación de datos. Los estudios nacionales tratan del estrés (Inventario de Síntomas de Estrés para Adultos de Lipp), condiciones psíquicas (Escala de Autoestima de Rosemberg, SRQ-20, Escala de Apoyo Social, SRQ), calidad de vida (WHOQOLBref), clima organizacional (Diagnóstico Organizacional de Krausz), nivel de actividad física (IPAQ), alcoholismo (CAGE Test) y condiciones económicas (CCEB). Los estudios internacionales tratan sobre instrumentos de transtornos relacionados a eventos traumáticos (Traumatic Stress Schedule, Impact of Event Scale, Critical Incident History Questionnaire, Life Stress Checklist - Revised, Posttraumatic Stress Disorders Checklist-specific, Peritraumatic Distress Inventory), así como de instrumentos relacionados con temáticas de condiciones de estrés, como depresión (Hamilton Depression Rate Scale, The Beck Depression Inventory), alcoholismo (Michigan Alcohol Screening), resistencia (Checklist Individual Strength), histórico familiar (Family History Screen), ajuste social (Social Adjustment Scale Self Report), y diversas otras condiciones psiquiátricas (Hopkins Symptom Checklist-21, Self Report Questionnaire; 90-item Symptom Checklist, 
International Classification of Primary Care, Structured Clinical Interview for DSM-IV, Global Symptom Index), físicas (Physical Conditions Test) y ocupacionales (Occupational Stress Index, Mississippi Combat Scale Civilian Version). Además del uso de cuestionarios estandarizados, gran parte de los estudios también han adoptado instrumentos especialmente construidos para esa investigación ( $\mathrm{f}=3$ ).

\section{Discusión}

El mayor número de publicaciones sobre el nivel de actividad física y los funcionarios de salud en Brasil fueron en la región sureste, y ésto puede estar directamente relacionado con un mayor número de universidades y cursos de postgrado en diversas áreas del conocimiento humano.

Según la literatura especializada, actualmente la región Sureste cuenta con casi la mitad de la población $(42 \%)$, la mayor parte de la riqueza nacional medida por el producto interno bruto $(70 \%)$, tecnología de producción $(84 \%)$ de la producción científica $(79 \%)$, los investigadores $(69 \%)$ que cubre más de la mitad de las publicaciones (18-20). De manera polémica, los estudios indican que la región sureste también tiene uno de los más importantes contingentes pobres del país y uno de los más altos índices de criminalidad y violencia urbana que afectan a la seguridad y la salud pública, y como consecuencia afectando la salud de los policías $(36,37)$.

La preponderancia de estudios internacionales sobre la sociedad estadounidense, también puede ser relacionado la verificación de que Estados Unidos posee casi la mitad de las mejores universidades del mundo, lo que favorece fuertemente a la producción académica promoviendo la investigación científica y la modificación social. Estados Unidos, todavía posee una fuerte comunidad académica en el área de justicia criminal, la cual proporciona esencialmente el soporte cognitivo en función de los asuntos relativos a la formación, entrenamiento y extensión a la investigación en esa importante área del Estado (38).

La mayor cantidad de publicaciones sobre agentes de seguridad publica en Brasil, fue en la ciudad de Rio de Janeiro, una de las mayores ciudades de la región sudeste. El conglomerado de personas en estas macrometrópolis aumenta la criminalidad y violencia urbana. Los datos estadísticos apuntan que $90 \%$ de las muertes violentas en Río de Janeiro son cometidas por el uso de armas de fuego y el $70 \%$ de los homicidios son provocados por enfrentamientos entre los traficantes $y$ los traficantes con los policías, afectando de este modo, la salud de éstos y tornándose un campo provechoso para estudios científicos (39).

A pesar de los criterios de inclusión de estudios solamente con policías militares fueron encontrados otros estudios con diferentes tipos de agentes respectivamente, como por ejemplo: fuerzas armadas, policía federal, policía civil y policía militar, entre otros, llevando a pensar acerca de las diferentes actuaciones en seguridad publica previsto en la legislación brasilera, ya que las fuerzas pueden, en casos determinados por el Gobierno Federal, actuar de manera activa en el combate de la criminalidad. En cuanto la Policía Federal actúa investigando los crímenes federales y vigilando las fronteras, la Policía Civil presenta una función investigativa actuando en conjunto con la justicia y el Ministerio Publico; y la Policía Militar hace la actuación ostensiva y preventiva, lo que hace pensar a un mayor cuadro de servidores en relación con los otros policías $(40,41)$. A pesar de la gran cantidad de agentes de seguridad publica, el Ministerio de Justicia (42), destaca que este cuadro no ha acompañado el crecimiento poblacional de diversas regiones de Brasil, lo que ha llevado a tener diversos problemas de salud entre la policía, debido a su actuación directa con la criminalidad en defensa del ciudadano.

Considerando que los estudios nacionales analizados presentan como motivación principal las investigaciones aplicadas sobre problemas en el área de la salud y actividad física policial, principalmente con delineamientos cualitativos provenientes de la psicología. Ésto se puede comprender al constatar que esta área científica y profesional estudia el comportamiento y los procesos mentales como emociones, atención, memoria y análisis de las reacciones del ser humano frente a las diversas situaciones enfrentadas y que en el caso de los agentes de seguridad publica, directamente relacionados a los estados de estrés, salud mental, nivel de actividad física, calidad de vida, enfermedades físicas y mentales, y dependencia química, entre otras reacciones típicas de ocupaciones expuestas a situaciones de conflicto y de extrema presión individual y colectiva (43). Mas allá de diferentes factores, tales como exposición diaria a eventos traumáticos, sistemas organizacionales con jerarquías rígidas, largas jornadas de trabajo y baja renta mensual, entre otros $(8,23,44)$. 
Contrariamente a los estudios nacionales, los estudios internacionales son de abordaje cuantitativo, con objetivos clasificados como descriptivos y procedimientos metodológicos de levantamiento, tanto en secciones de temporalidad transversal y longitudinal, realizados por medio de instrumentos del área de trastornos de estrés relacionados con eventos traumáticos. Estudios apuntan que la profesión de los oficiales es considerada extremamente estresante según a Occupational Disease Intelligence Network (ODIN) Y la Occupational Stress and Mental lllness (SOSMI) $(45,46)$. Así, es posible señalar que esta condición se ha acentuado en las últimas décadas en diversos países de América del Norte, Europa, Asia y del Medio Oriente, en donde, las divergencias políticas, económicas, culturales y religiosas se han acentuado en un estado de extrema inestabilidad en el área de seguridad publica (47).

En segundo orden, se puede constatar que la revisión sistemática sobre la salud policial identificó un pequeño número de artículos seleccionados relacionados a la actividad física y al estado nutricional. En este sentido, a pesar de la prevalencia de las patologías psíquicas en la actuación de seguridad publica, se puede considerar una debilidad en las discusiones ocurridas en las décadas finales de este siglo sobre la salud pública que segmentaban y acentuaban las diferentes instancias del concepto de salud, pués no se puede ignorar que los agentes de seguridad pública, están expuestos a todas las patologías típicas de las sociedades modernas relacionadas con enfermedades crónicas degenerativas (39). En este sentido, se puede destacar que casi la mitad de la población de los países desarrollados, presentan exceso de peso corporal y parte considerable de las muertes en el mundo ocurren por enfermedades cardiovasculares, ambas causas y efecto del estilo de vida sedentario predominante en la actualidad (48-50).

Se observa un pequeño número de artículos publicados en Brasil sobre la salud, la actividad física y la inactividad entre los agentes de la policía, a pesar del gran número de artículos científicos publicados en forma de tesis, tesinas, la finalización de curso (la graduación y especialización) y también de otros agentes de la segurid3A pública, como las Fuerzas Armadas, de la Policía Civil, la Guardia Municipal, entre otros.

Sin embargo, hay que considerar que las corporaciones de policía militar en Brasil aún pueden considerarse conservadoras, lo que impide el acceso y la realización de investigación científica sólida. La Policía Militar tiene un mayor número de agentes en Brasil en comparación con otros tipos de policía, lo que puede explicar el mayor número de búsquedas con sus agentes, sobre todo porque se consideran como clase profesional que se enfrenta a diario a acontecimientos traumáticos, contactos directos con la violencia urbana, arriesgan su propia vida para defender al ciudadano y la equidad pública y sufren presiones psicológicas propias de jerarquía rígida, lo que dificulta la actividad física y conduce a trastornos psicológicos graves.

Los estudios exploran los pequeños diseños experimentales, tanto nacionales como internacionales, así como han dejado de lado la promoción y prevención de varias otras causas de las enfermedades asociadas con la policía, tales como la inactividad física, la obesidad y las enfermedades cardiovasculares.

Artículos nacionales se desarrollan principalmente a través de los enfoques cualitativos, e internacionales a través de métodos cuantitativos, pero ambos hacen hincapié en temas relacionados con la salud física y mental, en especial en relación con el estrés. En consecuencia, se sugiere una mayor apertura de los campos de la investigación científica, con el objetivo de contribuir a superar los problemas encontrados desde los municipios más pequeños a las grandes ciudades de Brasil, el foco de los principales núcleos de población y por lo tanto los graves problemas de seguridad pública. Todo ésto ocurre porque la delincuencia crece a medida que aumenta el poder económico, las desigualdades sociales y a pesar del creciente número de universidades y de estudios de posgrado hacen hincapíe en la producción de conocimiento científico acerca de la salud mental, la educación física y el nivel de actividad física de los agentes de seguridad pública, el número es todavía pequeño en comparación con muchos países desarrollados.

\section{Financiamiento}

Esta investigación recibió apoyo financiero de la Fundação de Amparo à Pesquisa de Santa Catarina (FAPESC).

\section{Referencias}

1. Andrade HC. Das medidas de segurança. [Tesis doctoral]. Faculdad de Direito, Universidad Federal de Minas Gerais, Belo Horizonte; 2001

2. Andrade ER, Sousa ER, Minayo MCS. Intervenção visando a auto-estima e qualidade de vida dos policiais civis do Rio de Janeiro. Ciênc. saúde coletiva. 2009; 14(1): 275-85. 
3. Andrade ER, Souza ER. Auto-estima como expressão de saúde mental e dispositivo de mudanças na cultura organizacional da polícia. Psic. Clin. 2010; 22(2): 179-95.

4. Minayo MCS, Assis SG, Oliveira RVC. Impacto das atividades profissionais na saúde física e mental dos policiais civis e militares do Rio de Janeiro (RJ, Brasil). Ciênc. saúde coletiva. 2011; 16(4): 2199-209.

5. Kamble SV, Phalke DB. Study of occupational stress as a risk factor for various morbidities among policemen. J Indian Med Assoc. 2011; 109(4): 238-40.

6. Sorensen L, Smolander J, Louhevaara V, Korhonen O, Oja P. Physical activity, fitness and body composition of finnish police officers: a 15-year follow-up study. Occup. Med. 1999; 50: 3-10.

7. Gonçalves OGL. Aptidão física relacionada à saúde de policiais militares do município de Porto Velho - RO. [Disertación de Maestría]. Universidad de Brasília, Brasília; 2006.

8. Leino T, Selin R, Summala H, Virtanen M. Work-related violence against security guard - who is most at risk? Ind Health. 2010; 49(2): $143-50$

9. Lopes CL. Caracterização dos acidentes de trabalho dos bombeiros militares de santa e polícia militar de Santa Catarina. [Monografia de Especialización en Ingeniería de Seguridad Ocupacional]. Universidad Del Sul de Santa Catarina: Palhoça; 2009.

10. Minayo SCM, Souza RE, Constantino P. Riscos percebidos e vitimização de policiais civis e militares na (in) segurança pública. Cad. Saúde Pública. 2007; 23(11): 2767-779.

11. Oliveira EAM. Validade do teste de aptidão física do Exército Brasileiro como instrumento para a determinação das valências necessárias ao militar. Revista de Educação Física. 2005; 131: 30-8.

12. Spode CB, Merlo ARC. Trabalho policial e saúde mental: uma pesquisa junto aos capitães da polícia militar. Psicol. Refl. Crít. 2006; 19(3): 362-70.

13. Costa A, Grossi B. Relações intergovernamentais e segurança pública: uma análise do fundo nacional de segurança pública. Revista Brasileira de Segurança Pública. 2007; 1(2): 6-20.

14. Silva MB, Vieira SB. O processo de trabalho do militar estadual e a saúde mental. Saude Soc. 2008; 17(4): 161-70.

15. Oliveira LK, Santos ML. Percepção da saúde mental em policiais militares da força tática e de rua. Sociologias. 2010: 12: 224250.

16. Jesus MG, Jesus AFE. Predisposição para desenvolver resistência insulínica em policiais militares. Pensar a Prática. 2010; 13(2): 1-15.

17. Souza ER, Franco LG, Meireles CC, Ferreira VT, Santos NC. Sofrimento psíquico entre policiais civis: uma análise sob a ótica de gênero. Cad. Saúde Pública. 2007; 23(1): 105-14.

18. Costa M, Accioly Junior H, Oliveira J, Maia E. Estresse: diagnóstico dos policiais militares em uma cidade brasileira. Pan Am J Public Health. 2007; 4(21).

19. Ferreira SKD, Bonfim C, Augusto SGL. Fatores Associados ao Estilo de vida de Policiais Militares. Ciênc. Saúde coletiva. 2011; 16(8).

20. Anchieta VCC, Galinkin AL, Mendes AMB, Neiva ER. Trabalho $e$ riscos de adoecimento: um estudo entre policiais civis. Psic. Teor. e Pesq. 2011; 27(2)

21. Nabeel I, Baker BA, McGrail Junior MP, Flottemesch TJ. Correlation between physical activity, fitness, and musculoskeletal injuries in police officers. Minnesota Medicine, New York. 2007; 90(9): 40-43.

22. Charles LE, Fekedulegn D, McCall T, Burchfiel CM, Andrew ME, Violanti JM. Obesity, White Blood Cell Counts, and Platelet Counts among Police Officers. Obesity. 2007; 15(11).
23. Huddleston LM, Paton D, Stephens C. Conceptualizing traumatic stress in police officers: Pre-employment, critical incident and organizational influences. Traumatology. 2006; 12(3): 170-7.

24. Slottje P, Witteveen AB, Twisk JW, Smidt N, Huizink AC, Mechelen $\mathrm{W}$ et al. Post-disaster physical symptoms of firefighters and police officers: Role of types of exposure and post-traumatic stress symptoms. British Journal of Health Psychology. 2008; 13: 327-342

25. Strating M. A job-related fitness test for the Dutch police. Occupational Medicine. 2010; 60: 255-260.

26. Geldman PM. The effect of pre-injury physical fitness on the initial severity and recovery from whiplash injury, at six-month follow-up. Clinical Rehabilitation. 2008; 22: 364-376.

27. Inslicht SS. Family Psychiatric History, Peritraumatic Reactivity, and Posttraumatic Stress Symptoms: A Prospective Study of Police. J Psychiatr Res. 2010; 44(1): 22.

28. Kapusta ND, Voracek M, Etzersdorfer E, Niederkrotenthaler T, Dervic K, Plener PL et al. Characteristics of Police Officer Suicides in the Federal Austrian Police Corps. Crisis. 31(5):265-271, 2010.

29. Mclean N, Marshall LA. A front line police perspective of mental health issues and services. Criminal Behaviour and Mental Health. 2010; 20: 62-71.

30. Rajaratnam SM, Barger LK, Lockley SW, Shea SA, Wang W, Landrigan CP et al. Sleep Disorders, Health, and Safety in Police Officers. JAMA. December, 2011; 21(306): 23.

31. Kesic D, Thomas SDM, Ogloff JRP. Mental illness among police fatalities in Victoria 1982-2007: case linkage study. Australian and New Zealand Journal of Psychiatry. 2010; 44: 463-468.

32. Komarovskaya I, Maquen S, McCaslin SE, Metzler TJ, Madan $\mathrm{A}$, Brown $\mathrm{AD}$ et al. The impact of killing and injuring others on mental health symptoms among police officers. I. Journal of Psychiatric Research. 2011; 45: (332-336).

33. Ramey SL, Dowming NR, Knoblauch A. Developing Strategic Interventions to Reduce Cardiovascular Disease Risk Among Law Enforcement Officers. AAOHN Journal, February. 2008; 56(2).

34. Sassen B, Cornelissen VA, Kiers H, Wittink H, Kok G, Vanhees L. Physical fitness matters more than physical activity in controlling cardiovascular disease risk factors. European Journal of Cardiovascular Prevention and Rehabilitation. 2009; 16(6).

35. Gil AC. Métodos e técnicas em pesquisa social. São Paulo: Atlas; 1999.

36. Peixoto BT, Lima RS, Durante MO. Metodologias e criminalidade violenta no Brasil. São Paulo Perspec. 2004; 18(1): 13-21.

37. Albuquerque E M, Simões R, Baessa A, Campolina B, Leandro L. A distribuição espacial da produção científica e tecnológica brasileira: uma descrição de estatísticas de produção local de patentes e artigos científicos. Revista Brasileira de Inovação. 2002;1(2): 225-51.

38. Dantas LFG. Cooperação bilateral Brasil/EUA na área policial: histórico, perspectivas e possibilidade. Polícia e Segurança Pública. 2012 [Acceso 22 Mar 2012]. Disponible en: http://www. policiaeseguranca.com.br/bilateral.htm.

39. Souza ER, Minayo MCS. Policial, risco como profissão: morbimortalidade vinculada ao trabalho. Ciênc. Saúde Coletiva. 2005; 10(4): 917-28.

40. Brasil. Constituição de Brasília: Senado Federal, Centro Gráfico, 1998.

41. Pate RR, Pratt M, Blair SN, Haskell WL, Macera CA, Bouchard $\mathrm{C}$ et al. Physical activity and public health: a recommendation from the centers for disease control and prevention and the American College of Sports Medicine. JAMA, 1995; 273(5): 402-7. 
42. Ministério da Justiça. Portal do Cidadão. 2012 [Acceso: 23 Mar 2012]. Disponible en: http://portal.mj.gov.br/data/Pages/ MJA21B014BPTBRIE.htm.

43. Neves I. Direito da segurança social, princípios fundamentais: uma análise prospectiva. Coimbra: Coimbra; 1996.

44. Schlichting Junior AM, Silva R. Revisão sistemática da produção científica relacionada à atividade física e qualidade de vida de militares. Revista de educação física do Exército. 2009; 145(3): 28-36.

45. Collins PA, Gibbs CC. Stress in Police Officers: a study of the origins, prevalence and severity of stress-related symptoms within a county police force. Occupational Medicine. 2003; 53(4): 256-64.
46. Darling CA, Hill EW, Mcwey LM. Understanding stress and quality of life for clergy and clergy spouses. Stress and Health. 2004; 20: 261-77.

47. Wieviorka M. A democracia contemplativa. Instituto Humanitas. 2012 [Acceso: 23 Mar 2012]. Disponible en: http://www. ihuonline.unisinos.br/index.php?option $=$ com busca

48. Pollock ML, Wilmore JH. Exercícios na saúde e na doença: avaliação e prescrição para prevenção e reabilitação. São Paulo: MEDSI; 1993.

49. Blair SN, Applegate WB, Dunn AL, Ettinger WH, Haskell WL, King AC et al. The Activity Counseling Trial Research Group Activity Counseling Trial: rationale, design, and methods. Med Sci Sports Exerc. 1998; 30(7): 1097-106.

50. Paffenbarger RS, Lee IM. Physical activity and fitness for health and longevity. Res. Q. Exerc. Sport. 1996; 67(3): S11-28. 